\title{
Die Präsenz Theodor Kochers im Werk des Triestiner Chirurgen Gustavo Usiglio über die Schilddrüsentumoren (1894)
}

von Loris Premuda

ZuSAMmenfassung

Obwohl von den Medizinhistorikern bisher nicht zur Kenntnis genommen, pflegte Gustavo Usiglio (1855-1933) in Triest eine Chirurgie von hohem Rang. Er war Schüler Billroths und widmete diesem seine Monographie über Blasenstein-Zertrümmerung (1882).

In seinem hier vorgestellten Werk über Schilddrüsen-Geschwülste - im wesentlichen geht es um den Kropf - zeigt sich Usiglio als begeisterter Jünger Kochers.

\section{Der Autor}

Gustavo Usiglio ist ein Chirurg, dessen Name bis jetzt in keinem Handbuch oder Traktat über Geschichte der Medizin aufscheint ${ }^{1}$. Die historische Erfahrung lehrt uns, dass es im Bereich der Medizin mehr als einen miles ignotus gibt, der in Theorie oder Praxis aktiv tätig war, aber dessen Werk aus vielleicht verständlichen Gründen oder aufgrund des ausbleibenden Erfolgs in Vergessenheit geraten ist. Usiglio gehört meiner Meinung nach dieser letzten Kategorie an.

Er wurde in Triest am 1. März 1855 geboren und besuchte hier das Gymnasium, wo er die Matura am 31. Juli 1873 ablegte. Er studierte sechs Semester (1873-76) an der Universität Graz und vier Semester (1876-78) an der Universität Wien, anschliessend wieder in $\mathrm{Graz}^{2}$ und promovierte zum Doktor der gesamten Heilkunde am 25. Juli $1879^{3}$. Daraufhin besuchte er die II. Chirurgische Klinik in Wien unter Billroth ${ }^{4}$ und war dort Operationszögling ${ }^{5}$. Nach einem dreijährigen Aufenthalt in Wien zieht er nach Triest und wird in kurzer Zeit Erster Assistent beim Chirurgen Theodor Escher (1847-1923), einem ehemaligen Assistenten von Rose in Zürich und Billroth in Wien. Anschliessend wurde Escher Chefarzt der IV. Chirurgischen Abtei- 


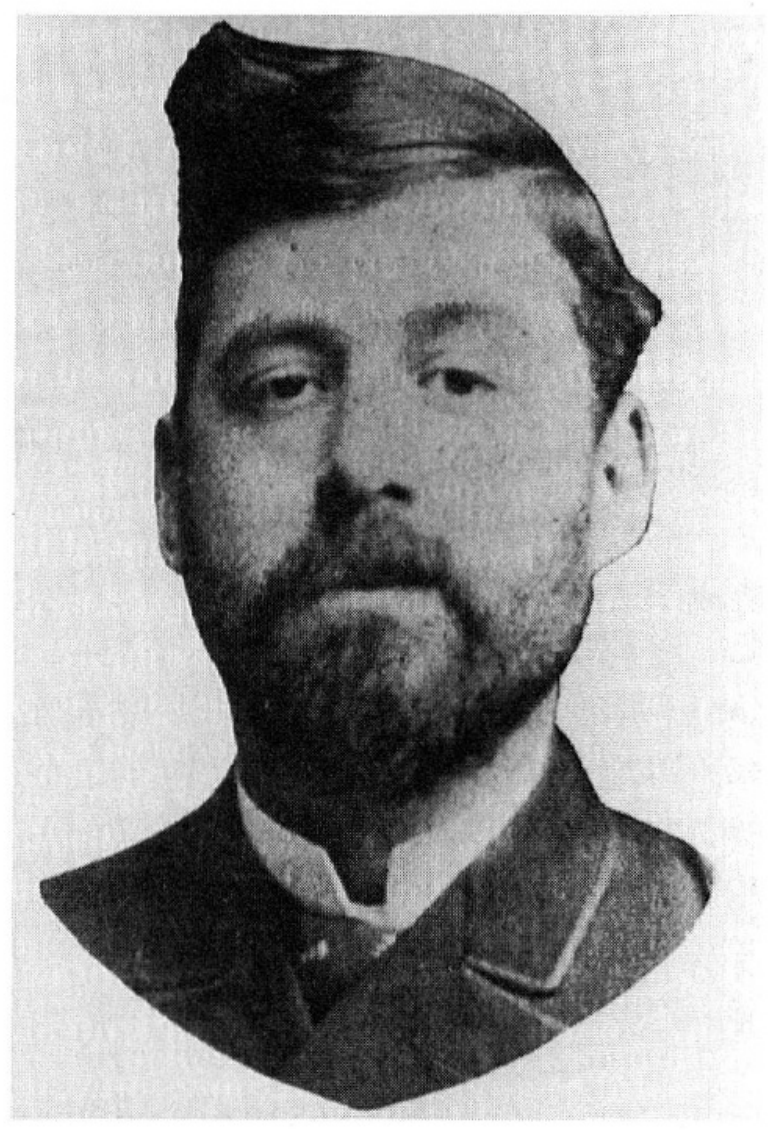

Abb.1: Gustavo Usiglio (1855-1933).

lung des Städtischen Krankenhauses von Triest, «das, was seinen wissenschaftlichen Wert und seine zahlenmässige Leistungsfähigkeit betrifft, gleich nach den Wiener Krankenhäusern kommt» ${ }^{6}$. In dem Buch, das Gegenstand dieses Vortrages ist ${ }^{7}$, wird Escher von Usiglio als «mein Lehrer und Freund» bezeichnet ${ }^{8}$.

Usiglio war Autor einiger Dutzend Monographien und Artikel von chirurgischem ${ }^{9}$ und urologischem Inhalt ${ }^{10}$. Nach ungefähr zwölf Jahren zog er sich von seiner Tätigkeit im Krankenhaus zurück und war politisch im liberalnationalen Feld tätig. Er war Arzt in einem kommunalen Sanitätsbezirk. Die letzten Jahre seines Lebens verbrachte er in einer kleinen Villa am (damaligen) Stadtrand von Triest und widmete sich der Gärtnerei. Der Tod überraschte ihn am 25. Januar $1933^{11}$.

Der Autor dieses kurzen Vortrags hegt auch heute noch Zweifel und Bedenken, die er nicht mit Sicherheit auszuräumen vermag, was wohl die Gründe und Faktoren waren, die dem vielversprechenden, aus einer hervor- 
ragenden Schule kommenden Chirurgen eine glänzendere Karriere verwehrten. Vermutlich haben Charakterzüge oder depressive Elemente, mangelndes Selbstvertrauen oder Neid mitgespielt und seinen als sicher geltenden Aufstieg zu höheren Zielen behindert.

Für die Kropfproblematik zeigte Usiglio schon im Jahre 1881 Interesse, als in Billroths Klinik, in seiner Anwesenheit, von der Stirn eines 50jährigen Patienten ein Tumor entfernt wurde, der sich infolge der histologischen Untersuchung, durchgeführt von seinem Freund Dr. Ehrendorfer (später Professor in Innsbruck), als kropfähnliches Gewebe erwies. Usiglio schreibt, bestärkt durch die Meinung der Vorgesetzten, dass es sich vermutlich um einen «metastasischen Kropf der Stirn» gehandelt habe ${ }^{12}$. Bereits 1884 veröffentlichte Usiglio, als Assistent im Krankenhaus von Triest, ein «Originalmemoire» über «L'estirpazione del gozzo, con un caso eseguito a mezzo del laccio emostatico» (Die Kropfexstirpation, mit einem mittels hämostatischen Gummischlauch durchgeführten Fall) ${ }^{13}$.

\section{Das Buch}

Das Werk «Sui tumori della tiroide e loro cura» umfasst 148 Seiten. Es enthält eine in 19 Kapitel aufgeteilte monographische Darstellung des gesamten Problems und anschliessend ein 30 Seiten langes Kapitel zum therapeutischen Aspekt. Der Autor erweist sich in bibliographischer Hinsicht bestens informiert und als Träger einer soliden Bildung, auch in den basic sciences. Die zur Aufgliederung des Werkes angewandte Methode ist eindeutig wissenschaftlicher Natur - wie übrigens in allen anderen Werken der Schule Billroths -, obwohl es erst veröffentlicht wurde, als der Autor die Klinik bereits seit mehr als zehn Jahren verlassen hatte. Sein Ansatz bei der Erörterung des medizinisch-chirurgischen Themas ist selbstverständlich unverändert geblieben.

Es steht fest, dass Usiglio Kochers spezifische wissenschaftliche Produktion kannte und aufmerksam verfolgte, denn er selbst schreibt, dem Berner Kliniker für die Übersendung seines «Berichtes über weitere 250 Kropfexstirpationen» gedankt zu haben, die 1889 im «Correspondenz-Blatt für Schweizer Ärzte» erschienen war ${ }^{14}$.

Zur Erforschung der Schilddrüsenerkrankungen wurde der Triestiner Chirurg anscheinend vorrangig durch eine von Kocher noch im Jahre 1878 als Antwort auf drei Fragen formulierte Behauptung angeregt ${ }^{15}$ : 


\section{SUI TUMORI DELLA TIROIDE}

\section{F IORO OURA}

DEL.

\section{Dott. GUSTAYO USIGLIO}

"ia Oneratore nella Cliniea del Prof. Billroth di Vienna

- primo Chirurgo Assistente nell Ospedale civico di Trieste

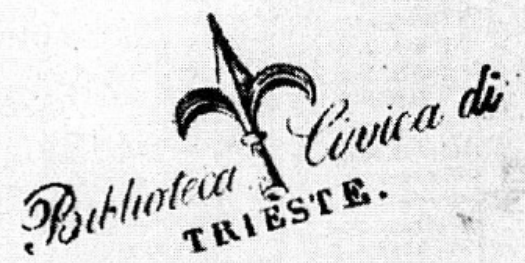

\section{CASA EDITRICE}

\section{DOTTOR FRANCESCO VALLARDI}

MILANO, Corso Magenta, 48. NAPOL, S. Anna dei Lombardi, 36. TORINO FIRENZE

PADOVA - PIS I - PAVIA - PALERMO - CATANIA

Abb.2: Titelblatt des Buches von Gustavo Usiglio. 
«Es fragt sich vielmehr: Sind auch hier analoge minimale corpusculäre Entzündungserreger im Spiele? Gelangen sie auch hier von Aussen in den Körper? Und auf welchem Wege? Antworten auf diese Frage glaube ich einer längeren Beobachtung und experimentellen Prüfung zweier Krankheitsformen entnehmen zu können, welche in Bern relativ häufig zur Beobachtung kommen, nämlich der acuten Osteomyelitis und der acuten Strumitis.» ${ }^{15}$

Sicher ermutigend konnten für Usiglio die für die damalige Epoche (1883) äusserst positiven Behauptungen klingen, die Kocher in seiner grundlegenden Monographie zum Ausdruck bringt ${ }^{16}$ :

«Ich habe bis zur Stunde 101 Mal die Kropfexstirpation ausgeführt. Von diesen 101 sind 13 Patienten gestorben, entsprechend einer Mortalität von 12,8 pCt.»

Meisterhafte Ergebnisse angesichts der reichen Statistik im Vergleich zur vorhergehenden Epoche ${ }^{17}$ :

«Während vor 1850 etwa 70 Kropfexstirpationen bekannt geworden sind, beläuft sich deren Zahl bis 1877 auf 146.»

Schon diese Zahlen reichen aus, um das überaus positive Urteil von Walter von Brunn zu rechtfertigen ${ }^{18}$ :

«In aller Erinnerung steht lebhaft noch das Gedächtnis des grossen Schweizers Theodor Kocher (1841-1917), Schülers von Langenbeck und Billroth. Sein Name bleibt unauslöschlich in den Annalen der Chirurgie verzeichnet als des bedeutendsten Kenners der Schilddrüsenaffektionen und ihres besten Operateurs.»

Wir dringen nun in die Seiten von Usiglios Buch vor, dessen Drucklegung fast hundert Jahre zurückliegt, um Ausmass und Inhalt der Bezugnahmen auf Kochers Werk zu unterstreichen.

Im 48 Seiten langen Vorwort des Werkes sind Angaben über Anatomie, Embryologie, Physiologie, Physiopathologie und Transplantationen der Schilddrüse enthalten. In den darauffolgenden 60 Seiten befasst sich der Autor mit dem onkologischen Problem sensu lato: Kröpfe und bösartige Tumoren. Die letzten 30 Seiten, die am stärksten von eigenen Beobachtungen geprägt sind, behandeln die Therapie in medizinischer und chirurgischer Hinsicht.

Es erübrigt sich hier hervorzuheben, dass das Myxödem, d.h. das paradigmatische Syndrom der Schilddrüseninsuffizienz auf verschiedene Ursachen zurückzuführen ist. Unter anderem verursacht die chirurgische Totalexstirpation des Kropfes ein postoperatives Myxödem. Wenn diese Definition dem Genfer Chirurgen Jaques-Louis Reverdin (1842-1929) zuzuschreiben ist, so stammt die folgende, Cachexia strumipriva, von Kocher, der als feiner 
klinischer Beobachter bereits «im denkwürdigen Jahre 1883» in jener Antwort an Ord über die Kachexie geschrieben hatte ${ }^{19}$ :

«Ich bin einverstanden, dass die Verbindung eines Schilddrüsenleidens mit einer Erregung des sympathischen Nervensystems bei der Basedowschen Krankheit einen wohl an eine Analogie im gegenteiligen Sinn denken lässt.»

Usiglio hält aufgrund seiner Erfahrung die beiden Definitionen von Reverdin und von Kocher für Synonyme ${ }^{20}$.

Ausserdem betont und bejaht er Wölflers Erklärung, dass Billroth, im Unterschied zu Kocher, keine Fälle von Cachexia strumipriva im II. und III. Stadium beobachtet habe. Die bedeutendsten klinischen Phänomene nervöser Natur waren vom Wiener Kliniker nicht beobachtet worden, da sich seine Patienten frühzeitig der nachfolgenden Kontrolle entzogen hatten ${ }^{21}$.

Der Triestiner Chirurg unterstreicht die von Reverdin und Kocher anerkannte enge Verbindung zwischen Kropf und Kretinismus, in Einklang mit dem von einer sardischen Ärztekommission hervorgehobenen Phänomen. Nach der Überzeugung dieser Kommission wird die Hälfte der an Kretinismus leidenden Kinder von kropfigen Eltern geboren ${ }^{22}$. Ferner betont er die Gewichtigkeit der Beiträge Reverdins und Kochers zur «genauen Kenntnis des postoperativen Myxödems beim Menschen» ${ }^{23}$ und präzisiert, dass für den Berner Chirurgen das Myxödem eine unheilbare Krankheit der Jugendlichen ist ${ }^{24}$.

Historisch gesehen, ist es von grösster Bedeutung, dass

«Anfangs der 80er Jahre von chirurgischer Seite, insbesondere von dem Berner Chirurgen Theodor Kocher, auf die Ausfallserscheinungen aufmerksam gemacht wurde, die nach Totalexstirpation der Schilddrüse auftraten und sich in kretinismusähnlichen Zuständen, bei Jugendlichen auch in Wachstumsstörungen, zeigten» ${ }^{25}$.

Auf das Jahr 1884 geht die Nachricht über das Experiment zurück, das der Physiologe Moritz Schiff, ebenfalls unter dem Ansporn der grundlegenden Beobachtungen des Berner Chirurgen, erfolgreich durchgeführt hatte; dabei wurde in die Bauchhöhle eines seiner Thyreoidea beraubten Hundes die Schilddrüse eines anderen Hundes eingepflanzt. Die Idee ist berauschend, und Usiglio macht sie zum Gegenstand einer intelligenten bibliographischen Untersuchung. Er berichtet über die Schilddrüsentransplantationen, die subkutanen und endovenösen Injektionen von Schilddrüsensaft, die Bluttransfusionen sowie über die Behandlung nach Howitz (1828-1912) und bringt auch die Angaben über die im Triestiner Krankenhaus im spezifischen Fall nach letzterer Methode gesammelten Erfahrungen mit ein ${ }^{26}$. 
Im letzten, nun im Mittelpunkt unserer Betrachtungen stehenden Kapitel des Buches, explodiert, wenn wir es so ausdrücken dürfen, die Macht der wissenschaftlichen Persönlichkeit Kochers, der als Erforscher jedes einzelnen Aspekts der Problematik der Schilddrüsenchirurgie, als gewandter und vortrefflicher Ausführer jedes Eingriffes und als scharfsinniger Kritiker jeder Operationstechnik in Erscheinung tritt.

Im Bereich der chirurgischen Therapie der Schilddrüsentumoren erkennt Usiglio fünf Methoden an: die Kauterisation, das Haarseil, die Inzision, die Exstirpation und die Unterbindung der Schilddrüsenarterien. Während die ersten beiden nach Meinung des Autors nur noch historische Bedeutung haben, wird die Exstirpation mit drei verschiedenen Methoden durchgeführt: Teilexstirpation, Enukleation (das sogenannte Evidement) und Resektion. Kocher erweist sich in jeder Operationstechnik als Meister. Usiglio greift den Grundgedanken des Berner Chirurgen auf, weshalb

«die Behauptung, dass die Exzision eines gewöhnlichen Kropfes, auch unter den schwierigsten Bedingungen, unabhängig von seiner Grösse und vom Alter des Patienten, nunmehr einen gefahrlosen Eingriff darstellt, nicht übertrieben erscheint, und es nicht mehr notwendig ist, nach Kropfexstirpationen von Mortalität zu sprechen» ${ }^{27}$.

Die allgemein durchgeführte antiseptische Behandlung und die verbesserten Techniken der Blutstillung sind die wesentlichen, für den Erfolg verantwortlichen Faktoren ${ }^{28}$.

Der Triestiner Chirurg beschreibt verschiedene Techniken, erläutert aber für die Strumektomie eingehend die von Kocher angewandte Methode, nach der der Kropf zunächst durch eine eckförmige, spezifische Merkmale aufweisende Inzision freigelegt wird. In der zweiten Phase des Eingriffes werden die grossen Arterien und Venen der Schilddrüse ausfindig gemacht. Hier möchte ich lediglich die operativen Ausführungen des Berner Chirurgen hervorheben, die Usiglio, Bezug nehmend auf Kochers Arbeiten von 1883 und 1889, betont: Es ist nicht notwendig, den N. recurrens aufzusuchen, um ihn nicht zu durchtrennen; es ist jedoch grösste Vorsicht geboten beim Entfernen der hinteren Teile des Kropfes, des sogenannten Hilus, um die Verletzung eines verdächtigen, eventuell hervortretenden Stranges, eben des N. recurrens, zu vermeiden. Auch die Herzäste des Sympathikus müssen unverletzt bleiben; ihre Verletzung kann sich ergeben, wenn die Schilddrüsenarterie innerhalb der A. carotis communis unterbunden wird ${ }^{29}$.

Die von Kocher erarbeiteten und von Usiglio zusammengefassten Lehren über die chirurgische Kropfbehandlung können daher in drei Schwerpunkte aufgegliedert werden: 
1. In jedem Fall ist ein reichlicher Blutverlust zu vermeiden.

2. Die Verletzung des N. recurrens und der Herzäste des Sympathikus muss ebenfalls vermieden werden.

3. Einen Teil der Schilddrüse (d.h. des funktionsfähigen Organs) muss man erhalten und die Totalexstirpation des Organs vermeiden ${ }^{30}$.

Bei der Beschreibung der Enukleation oder Teilexzisionsmethode und des Evidement der adenomatösen Kropfknoten schildert Usiglio, mit seinem Übermass an Genauigkeit, die nationalistisch angehauchte Polemik zwischen Bottini aus Pavia und Kocher. Der Berner Chirurg macht das Evidement - so wird im Buch betont - zu einer eigenständigen Methode: Die Drüsenkapsel wird nicht zuerst gespalten (wie bei der Enukleation), um den Tumor zu entnehmen, sondern das Skalpell wird zugleich durch Kapsel und Tumor geführt, wobei letzterer in zwei Hälften geteilt wird, die unmittelbar entweder mit den Fingern entnommen oder ausgelöffelt werden ${ }^{31}$.

Bei der in einem Unterkapitel angesprochenen «Kropfresektion nach Mikulicz» erkennt Usiglio dessen Technik das Verdienst zu, dass sich dadurch die Verletzung des N. recurrens besser als bei der Exstirpation vermeiden lässt, doch er erwähnt sogleich Kochers Vorschlag der «ResektionEnukleation», einer Koppelung der beiden Methoden ${ }^{32}$. «Kochers Methode» der Kropfexstirpation bei gleichzeitiger Wahrung eines Schilddrüsenteils widmet Usiglio sieben inhaltsreiche Seiten ${ }^{33}$.

Die Schwerpunkte werden von Usiglio zusammengefasst : Er entnimmt Kochers Gedanken den Grundsatz, nach dem die Exstirpation bei den diffusen, die Enukleation hingegen bei den umschriebenen Erkrankungen des Organs angezeigt ist. Auf die Exstirpation soll daher bei bösartigen Schilddrüsentumoren, entzündeten Kröpfen und bei den sogenannten diffusen Hypertrophien der Schilddrüse zurückgegriffen werden. Die Exstirpation eines ganzen Lappens ist nicht angezeigt, wenn die andere Hälfte fehlt oder völlig degeneriert ist. Die Enukleation ist bei Vorliegen von Kropfzysten und grossen, isolierten, adenomatösen Knoten angezeigt. Gerade in diesem Zusammenhang hebt Kocher die Fälle hervor, in denen sich das Evidement besser eignet als die Enukleation.

Das Werk, insgesamt ein Loblied auf den Berner Chirurgen - der damals noch nicht Nobelpreisträger war -, auf seinen Wagemut und seine grundlegenden technisch-chirurgischen Innovationen, schliesst mit seinen wichtigen Bemerkungen über die Ergebnisse der chirurgischen Eingriffe bei bösartigen Schilddrüsentumoren, die sich folgendermassen zusammenfassen lassen: 
«Eine rechtzeitig gestellte Diagnose beeinflusst beachtlich die Prognose der tumorbedingten Strumektomie» ${ }^{34}$.

Der Verfasser dieser wenigen Seiten freut sich, zum Schluss mit erneuter Überzeugung den Gedanken wiederholen zu dürfen, der die hervorragende Biographie des «Cantore» der Werke und Tage Theodor Kochers krönt, seines Landsmanns Ulrich Tröhler ${ }^{35}$ :

«Zusammenfassend kann man feststellen, dass in jenen fünfzig Jahren von keinem einzelnen Forscher und Praktiker eine grössere Förderung der Schilddrüsenforschung ausgegangen ist als von Kocher. Stets waren seine Fragestellungen zuerst chirurgisch. Doch entpuppt sich für uns seine schliessliche Suche nach Überwindung des chirurgischen Eingriffs nur als scheinbar paradoxe, in Wahrheit aber zwingende Bilanz einer verschlungenen Entwicklung, die ihn mindestens ebenso bestimmte wie er sie. Das weite Spektrum aller bekannten Forschungsmethoden, die er gezielt und andere anspornend einzusetzen wusste, stempelt Kocher in dieser Entwicklung zum richtigen Mann am richtigen Ort zur richtigen Zeit.» 


\section{Anmerkungen}

1 Usiglio wurde bisher nur von L.Premuda erwähnt, in: Die vermittelnde Funktion von Triest für die Verbreitung des medizinischen Denkens der Wiener Schule in Italien, in: Wien und die Weltmedizin (hg. von E. Lesky), Wien-Köln-Graz 1974, 99-115 (hier: 99); Cento anni di chirurgia a Trieste, Trieste, 1975, S. 31 (hier: 11).

2 Das erste medizinische Rigorosum legte Usiglio am 24. Juli 1876 mit «ausgezeichnetem» Erfolg, das zweite am 27. März 1879 mit «genügendem» Erfolg und das dritte am 23. Juli 1879 mit «ausgezeichnetem» Erfolg ab. Eine Dissertation war zur Erlangung des Doktorats nicht gefordert; man wurde nach der damaligen Studienordnung nach Absolvierung der Rigorosen zum Doktor promoviert. (Diese Angaben wurden mir freundlicherweise von Mag. Dr. Alois Kernbauer von der Universität Graz zur Verfügung gestellt, dem ich herzlich dafür danken möchte.)

3 Der Promotor für Gustavo Usiglio war Otto Rembold, Professor für Specielle medizinische Pathologie und Therapie. Vgl. zum Studienplan: F. von Krones, Geschichte der KarlFranzens-Universität in Graz, Graz 1886, 596-597.

4 Schon 1882 erwies Usiglio dem Lehrer seine Dankbarkeit, indem er ihm eine Monographie widmete, mit dem Titel «Litotrissia e litolapassia» (2. Ausgabe, Trieste 1888): «Al sommo maestro Teodoro Billroth con reverenza e gratitudine di discepolo».

5 Die Zentenarfeier des Operateur-Institutes der Wiener Med. Fakultät, Wien und Leipzig 1907, 83.

6 V.Cominotti, Premessa a: Resoconti sanitari dell'Ospedale Civico di Trieste per gli anni 1903-1908 pubblicati per cura del Dott. Vittorio Cominotti medico primario, Trieste 1912, S. 3.

7 G. Usiglio, Sui tumori della tiroide e loro cura, Milano 1894, S. 126. Hier bezieht man sich auf die 2. Ausgabe, Trieste (Balestra) 1895, S. 148.

8 Ib., 47.

9 Usiglio veröffentlichte 1881 die «traduzione con aggiunte autorizzate» del lavoro sul celebre intervento di Billroth con il titolo «Resezioni del piloro per carcinoma esposte dal Dott. Antonio Wölfler medico assistente nella Clinica Chirurgica del Prof. Billroth e docente di Chirurgia nella Università di Vienna», Bologna 1881.

10 Es ist zu berücksichtigen, dass Usiglio im Jahre 1880 in Wien auch Leopold von Dittel (1815-1898), Pionier der wissenschaftlichen Urologie in der Donaustadt, nahestand.

11 La morte del dott. Gustavo Usiglio, in: Il Piccolo, Trieste, giovedì 26 gennaio 1933.

12 G. Usiglio, L'estirpazione del gozzo, con un caso eseguito a mezzo del laccio emostatico, Gazzetta degli Ospitali V (1884) 36-37.

13 Ibid.

14 Op.cit. (s. Anm. 7), 128-129.

15 Th. Kocher, Zur Ätiologie der acuten Entzündungen, Archiv für klinische Chirurgie 23 (1878), 101-114 (hier: 101).

16 Ders., Über Kropfexstirpationen und ihre Folgen, ibid. 29 (1883), 254-337 (hier: 254).

17 Ibid.

18 W. von Brunn, Geschichte der Chirurgie, Berlin 1928, 296.

19 W.Tröhler, Der Nobelpreisträger Theodor Kocher, Basel 1984, 140.

20 Op.cit. (s. Anm. 7), 25. 
$21 \quad$ Ibid., 31.

22 Ibid.

23 Ibid., 33

24 Ibid., 34.

25 P. Diepgen, Geschichte der Medizin, II/2, Berlin 1955, 68.

26 Op. cit. (s. Anm. 7), 37-48.

27 Ibid., 129. Hier wurde das Zitat aus Usiglios Buch zurück übersetzt.

28 Ibid.

29 Ibid., 131-132.

$30 \quad$ Ibid., 136.

31 Ibid., 139.

32 Ibid., 141.

33 Ibid., 143.

$34 \quad$ Ibid., 147.

35 Op.cit. (s. Anm. 19), 156.

\section{Summary}

Theodor Kocher's presence in the work "On tumours of the thyroid" (1894) by the surgeon Gustavo Usiglio of Trieste

Gustavo Usiglio (1855-1933), a pupil of Billroth, was a surgeon of high qualities. The present author analyzes his main scientific work "On the tumours of the thyroid and their cure"; the book, which deals essentially with goitre, was published in 1894 at Milan and, in a $2^{\text {nd }}$ edition, in 1895 at Trieste. Especially in the final chapter on therapeutics, Usiglio reveals himself as a fervent follower of Kocher.

\section{Résumé}

La présence de Theodor Kocher dans le livre de Gustavo Usiglio, chirurgien à Trieste, «Sur les tumeurs de la thyroïde» (1894)

Gustavo Usiglio (1855-1933), élève de Billroth, pratiqua dans sa ville natale une chirurgie de haute valeur. Son livre «Sur les tumeurs de la thyroïde et leur traitement», analysé ici, fut publié en deux éditions (Milan 1894, Trieste 1895); il traite surtout du goitre. Usiglio s'y présente comme disciple fervent de Kocher. 


\section{Riassunto}

\section{La presenza di Theodor Kocher nell'opera «Sui tumori della tiroide» die Gustavo Usiglio, chirurgo triestino (1894)}

Gustavo Usiglio (1855-1933) è personaggio ignorato dai trattati di storia della medicina pur la sua attività scientifica e pratica in campo chirurgico è stata di buon livello. Nato a Trieste il $1^{\circ}$ marzo 1855 frequentò il «Ginnasio» della sua città e fu poi discepolo delle Università di Graz $\mathrm{e}$ Vienna. Conseguì la laurea a Graz e successivamente rimase tre anni nella Clinica Billroth a Vienna. Quindi passò come primo chirurgo assistente all'Ospedale Civico di Trieste sotto Theodor Escher (1847-1923), già assistente clinico di Rose a Zurigo e di Billroth a Vienna e poi primario nel capoluogo giuliano della IV Divisione Chirurgica.

Fin dal 1881 Usiglio è interessato al problema del gozzo, con un caso interessante di «gozzo metastatico della fronte». È inoltre autore di un libro «Sui tumori della tiroide e loro cura», che consta di 19 capitoli e di un capitolo alla fine di 30 pagine, che tratta l'aspetto terapeutico. Usiglio non conosce personalmente Theodor Kocher, dal quale però riceve l'omaggio di un fondamentale opuscolo sulla casistica di asportazioni della tiroide e del quale segue per buona parte principì e metodi con il grande rispetto di un discepolo. Il lavoro è costellato da acute e interessanti osservazioni, che mettono in luce la cultura medica e l'aggiornamento bibliografico dell'autore e la sua abilità chirurgica.

Prof. Dr. med. Loris Premuda

Istituto di Storia della Medicina

via Falloppio 50

I-35121 Padova 